\title{
Biopolítica y Ejemplaridad
}

\section{Biopolitics and Exemplarity}

Edgardo Castro ${ }^{1}$

\begin{abstract}
Resumen
El presente artículo explora, en relación con el concepto de biopolítica, la dimensión ontológico-metodológica de la noción de ejemplo. Desde esta perspectiva se ocupa del renovado interés por esta noción en el campo de la filosofía política contemporánea y se detiene, particularmente, en los análisis de Hannah Arendt y Martin Heidegger sobre la Retórica de Aristóteles. El autor propone la necesidad de una biopolítica de la ejemplaridad.
\end{abstract}

Palabras clave: Ejemplaridad. Biopolítica. Retórica.

\begin{abstract}
This paper analyses, in relation to the concept of biopolitics, the ontologicalmethodological dimension of the notion of example. From this perspective, it explores the renewed interest on this notion in contemporary political philosophy and particularly on the analyses of Hannah Arendt and Martin Heidegger about Aristotle's Rhetoric. It proposes a biopolitics of exemplarity.
\end{abstract}

Key words: Exemplarity. Biopolitics. Rethoric.

\footnotetext{
${ }^{1}$ Professor da Universidad Nacional de San Martín e Pesquisador Visitante CNPq na UFSC (Programa de Pós-graduação em Educação; Núcleo de Estudos e Pesquisas Educação e Sociedade Contemporânea). E-mail para contato: edgardomanuelcastro@gmail.com.
}

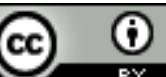

Esta obra foi licenciada com uma Licença Creative Commons - Atribuição 3.0 Não Adaptada. 


\section{Introducción}

Como hemos señalado en varias ocasiones, el término biopolítica, convertido en una palabra de moda, corre el riesgo de vaciarse de todo contenido específico (CASTRO, 2011, p.12). Ello se debe, por un lado, al hecho de que la pluralidad de enfoques posibles en torno a la cuestión del gobierno de la vida no ha sido acompañada de una discusión equivalente respecto de las cuestiones metodológicas. Se aceptan o rechazan conclusiones, tomándolas de un ámbito y transfiriéndolas a otro, pero sin plantearse cómo se llegó a ellas o si es posible proceder de este modo. Por otro lado, también han contribuido a esta deriva del término "biopolítica" las escasas indagaciones acerca de la historia conceptual de las categorías en torno a las cuales la corriente biopolítica ha tomado forma, como los propios conceptos de bíos y zoé.

Por varias razones, en el cruce de estos dos factores, el metodológico y el histórico-conceptual, nos encontramos con la noción de ejemplo. En primer lugar, porque ella se sitúa a la vez - como sucedía con la noción de discontinuidad, según el señalamiento de Foucault (1969, p. 17) - de los dos lados del problema: el de las herramientas conceptuales y el de los acontecimientos. La biopolítica puede proceder paradigmáticamente, mediante ejemplos, pero la vida en sí misma puede ser vida ejemplar. En segundo lugar, porque la noción de ejemplo nos permite abordar la cuestión que de algún modo anima, a modo de contrafigura, gran parte de la empresa biopolítica: la necesidad de pensar la vida más allá de la propia biopolítica, es decir, estableciendo con el lenguaje una relación que no pasa ni por la ley ni por la norma. Y, finalmente, en tercer lugar, porque la noción de ejemplo también nos permite vincular la cuestión metodológica de la biopolítica y sus conceptos con otras preocupaciones del pensamiento contemporáneo y, a través de ellas, con algunas cuestiones centrales de la tradición filosófica.

La tarea que nos proponemos en el presente trabajo es explorar, en relación con la problemática biopolítica, algunas de las proyecciones y de las raíces de la noción de ejemplo.

\section{Un renovado interés}

El griego "parádeigma" (compuesto por la preposición "pará" y el verbo 
"deíknynai", mostrar) ha sido traducido al latín por "exemplum", surgido del supino del verbo eximere (extraer, retirar). Tanto el griego "parádeigma" como el latín "exemplum" significan un caso particular (el que es exhibido o extraído) como la clase, el género o el modelo que es mostrado a través de él. "Paradigma" y "ejemplo" pueden tener, entonces, tanto un valor concreto como uno general, universal e incluso arquetípico. Ejemplificar consiste en elegir —con fines argumentativos, políticos o morales - el caso que remite a un conjunto de otros casos relativamente homogéneos, a una regla o a un ideal.

Respecto del vocabulario latino, como señala Agamben remitiendo al gramático Festo (s. II) (AGAMBEN, 2008a, p. 20), es necesario tener presente que, para dar cuenta de esta amplitud semántica que va del caso a la clase o al modelo, nos encontramos con la distinción entre exemplum y exemplar: « Ejemplo (exemplum) es lo que debemos seguir o imitar; ejemplar (exemplar), aquello de lo que debemos partir para hacer algo semejante. Lo primero se estima en el espíritu; lo segundo se ve con los ojos » (Festus, De significatione verborum).

En Signatura rerum. Sul metodo, Agamben hace de esta dualidad semántica del ejemplo la cuestión central del método o, más precisamente, de su método y del de Michel Foucault (AGAMBEN, 2008a, p. 33). Sus arqueologías son definidas, por ello, como investigaciones paradigmáticas, es decir, búsquedas del caso (el homo sacer, para Agamben, o el panóptico, para Foucault) que sirve como modelo de inteligibilidad (de la excepción soberana y del panoptismo, respectivamente). En este contexto metodológico, es necesario subrayarlo, Agamben sitúa el locus classicus de la noción metodológica de ejemplo en los Analíticos primeros de Aristóteles (AGAMBEN, 2008a, p. 20).

Junto a esta obra de Agamben, otros trabajos recientes también han hecho de la noción de ejemplo el eje de su exposición. Alessandro Ferrara en su The Force of the Example, se lamenta, en efecto, de la escasa fortuna que tuvo este concepto. Según sus propias palabras, se trata del destino de los third members (FERRARA, 2008, p. VII). Para remediarlo, situándose en la tradición kantiana de la Kritik der Urteilskraft, Ferrara se propone mostrar cómo la ejemplaridad, el juicio mediante ejemplos, puede constituirse en uno de los pivotes del pensamiento político contemporáneo, sobre todo, a la hora de superar los embates del denominado pensamiento postmoderno, que parecen haber minado la universalidad de los conceptos y de las teorías. 
También en relación con las posiciones postmodernas, pero en una perspectiva diferente, la relevancia del ejemplo es el tema central del libro de Irene Harvey, Labyrinths of Exemplarity. Pero Harvey no busca descubrir la fuerza del ejemplo a partir de Kant, sino de Heidegger y de Derrida o, más precisamente, del laberinto al que ellos conducen (HARVEY, 2002, p. X), para poder salir de él. Persiguiendo este objetivo, Harvey se remonta hasta la cuestión del ejemplo en la Retórica de Aristóteles, a la que le consagra una parte considerable del último capítulo de su investigación, aunque para distanciarse finalmente de él en términos críticos.

Más allá de la común referencia a la problemática de la ejemplaridad y de las relaciones que pueden establecerse entre sus recorridos argumentativos, ciertas diferencias aparecen, ya a primera vista, en estos trabajos. La primera y más notable, a nuestro modo de ver, concierne a las tradiciones conceptuales a las que cada uno de ellos remite de manera fundamental. Para Agamben, se trata del Aristóteles de los Analíticos primeros. Para Harvey se trata también de Aristóteles, pero no tanto el de los Análíticos, sino fundamentalmente del de la Retórica. Respecto de Ferrara, como señalamos, la referencia central es la Kritik der Urteilskraft de Kant. La segunda gran diferencia, concierne a la preocupación que guía cada una de estas investigaciones: el método de la arqueología filosófica, para Agamben; los límites de la deconstrucción, para Harvey; y la argumentación en el campo de la filosofía política, para Ferrara. Para Agamben, además, aunque no se detenga mayormente en ello, en la cuestión del ejemplo está en juego, principalmente, una ontología paradigmática (AGAMBEN, 2008a, p. 34).

Aristóteles y Kant aparecen, entonces, como el marco histórico-filosófico de este renovado interés por la noción de ejemplo. Aunque no de manera exclusiva (Agamben, de hecho, menciona, junto con Aristóteles, toda una serie de posiciones, desde Platón a Walter Benjamin), estos dos pensadores constituyen los puntos de apoyo del debate contemporáneo sobre el ejemplo.

Ahora bien, en la literatura filosófica del siglo XX, los textos de Aristóteles y Kant sobre el ejemplo han sido objeto de dos análisis particularmente significativos. No se trata, hablando con precisión, primariamente de libros, sino de lecciones que luego tomaron la forma de textos publicados: el seminario de Heidegger, del verano de 1924 (aparecido en el 2002, como el volumen XVIII de la Gesamtausgabe), donde se aborda en detalle e interpretativamente la concepción retórica de Aristóteles, y las lecciones del seminario de Hannah Arendt, del otoño de 1970 en la New School 
for Social Research (publicadas en 1982), sobre la Kant.

Una historia conceptual del ejemplo, desde la perspectiva del debate biopolítico contemporáneo, al menos a nuestro modo de ver, no puede no comenzar por estos dos trabajos; pues, en los cursos de Heidegger y Arendt, la cuestión del ejemplo y de la ejemplaridad se inscriben en el contexto del análisis de la estrecha relación entre vida, política y lenguaje que domina, precisamente, la discusión biopolítica.

\section{Una interpretación fenomenológica de la Retórica de Aristóteles}

Dos ambiciosos proyectos acompañaron los años de Heidegger en Marburgo, de 1923 a 1928, el que culminará con la publicación de Seind und Zeit en 1927 y el que, en cambio, nunca alcanzará su objetivo final, escribir un libro relevante sobre Aristóteles. A partir de 1925, en efecto, Heidegger se concentra en lo que se considera el primer esbozo de Sein und Zeit, los Prologomena zur Geschichte des Zeitbegriffes.

Durante estos años, si dejamos de lado el Natorp Bericht, escrito poco antes de llegar a Marburgo, Heidegger se ocupó considerable y minuciosamente de Aristóteles. $^{2}$ Sería erróneo, como afirma John Kress, suponer que ha sido simplemente casual, en relación con Sein und Zeit, este interés del joven Heidegger por los textos aristotélicos. Más bien todo lo contrario. Pero también sería un error pensar que en Sein und Zeit nos encontramos con una versión final y más desarrollada de la exégesis de los textos aristotélicos de los años marburgueses (KRESS, 2006, p. 329330).

Para expresarlo en pocas palabras, por un lado, a través del análisis fenomenológico del concepto griego y especialmente aristotélico de ousía, Heidegger es conducido hacia la centralidad de la cuestión de la temporalidad para abordar la cuestión del ser. La crítica del ser como presencia, ousía, constituye, de hecho, el punto de quiebre con la ontología clásica y de distanciamiento respecto de Aristóteles, cuya noción de temporalidad es, precisamente, objeto de crítica en el $\S 81$ de Sein und Zeit. Pero, por otro lado, en este giro hacia una concepción fenomenológica de la temporalidad y de la relación entre ser y tiempo, algunos conceptos centrales de los análisis marburgueses son, precisamente por ello, dejados de lado. En

\footnotetext{
${ }^{2}$ Para un balance detallado al respecto consultar Kisiel (1993).
} 
particular, la equivalencia entre vida fáctica (das faktisches Leben) y Dasein. En los trabajos marburgueses e inmediatamente anteriores, la estructura de la existencia, del Dasein, se corresponde con las estructuras de la vida fáctica. Ser es vivir. En Sein und Zeit, en cambio, la vida es excluida de la ontología fundamental de la existencia. Según la afirmación del propio Heidegger, "la vida no es ni un puro estar-ahí ni tampoco es un Dasein"; éste "nunca puede ser ontológicamente determinado como vida (ontológicamente indeterminada) y además otra cosa" (HEIDEGGER, 1967, p. 50). O, si queremos retomar las expresiones de la carta Ueber den Humanismus, no estamos en el mejor camino cuando definimos al hombre como un ser vivo (HElDEGGER, 2000, p. 15).

En el seminario de invierno de 1929-1930, poco tiempo después de la publicación de Sein und Zeit, titulado Die Grundbegriffe der Metaphysik, sobre el que se apoya parte de los análisis de G. Agamben en L'Aperto. L'uomo e l'animale (2002), Heidegger volverá sobre el problema de la vida y, más específicamente, sobre la vida animal. Pero lo hará en una perspectiva diferente a la explorada en los años marburgueses, donde la cuestión era abordada en el marco de una interpretación fenomenológica de la vida en términos prácticos (políticos, si queremos) y donde la Retórica de Aristóteles ocupaba un lugar central.

Ahora bien, la preocupación general de Heidegger en el curso de verano de 1924 es comprender algunos conceptos aristotélicos en su conceptualidad (Begrifflichkeit), es decir, situarse, de algún modo, en el suelo (Boden) en el que ellos han surgido y crecido. Es precisamente en la elucidación de la conceptualidad del concepto de lógos donde nos encontramos con el primer análisis de la fórmula aristotélica zôon lógon échon. Entre las múltiples posibilidades semánticas del término, según las consideraciones de Heidegger, lógos remite aquí al hablar, al légein. Lógos es el hablar de alguien con alguien o con otros acerca de algo y, por ello mismo, expresarse, escuchar y ser escuchado (HEIDEGGER, 2002, p. 20). No se trata, sostiene Heidegger, sólo de una determinación filosófica del hombre sino de una natürliche Vorfindlichkeit, es decir, de acuerdo con el vocabulario fenomenológico, de una posibilidad dada de antemano.

Del mismo modo que, según Heidegger, no podemos reducir esta experiencia del lógos a una determinación sólo filosófica del ser del hombre, tampoco podemos comprender lo que está en juego (la cosa misma) en el concepto de zoé (vida) a partir de la biología, la psicología o las ciencias sociales. Por ello, sostiene: "Zoé es un 
concepto del ser, 'vivir' remite a un modo del ser, en efecto, a un modo-de-ser-en-elmundo" (HEIDEGGER, 2002, p. 18).

En el segundo capítulo de la primera parte, Heidegger vuelve a plantearse en qué sentido debemos entender el término lógos y la expresión zôon lógon échon. Este capítulo se compone de cuatro largos parágrafos (§§ 9-12). En el primero de ellos, del que nos ocuparemos enseguida, Heidegger aborda la Política (libro I, cap. 2) y la Retórica (libro I, cap. 6) de Aristóteles. Zôon lógon échon y koinonía (comunidad) son los ejes temáticos de la exposición.

Todo el §9, con referencias alternadas a la Política y a la Retórica, se estructura en torno a la distinción, fundamental en el primero de estos tratados aristotélicos y a la que ya nos hemos referido, entre phoné y lógos, entre voz y discurso. Los objetivos fundamentales de la exposición de Heidegger son mostrar, por un lado, que se trata de dos modos del ser-en-el-mundo que definen a los seres vivientes y, por otro lado, de elucidar el modo de ser-en-el-mundo que está en juego en el lógos, en el discurso, como posibilidad co-originaria, en el ser del hombre, de su politicidad. Es en relación con este segundo objetivo donde la referencia a la Retórica se vuelve determinante.

La exposición de Heidegger comienza por la distinción entre zoé y bíos. Pero no se trata, en este contexto, de distinguir lo animal de lo humano; sino de las dos direcciones en que, en Aristóteles, puede llevarse a cabo "la clarificación de la estructura del ser-ahí (Dasein) del ser humano": zoé praktiké y bíos theoritikós, vida práctica y vida contemplativa (HEIDEGGER, 2002, p. 44). Ambas serán exploradas por Heidegger. Luego de esta observación, se pasa directamente al análisis de la distinción entre phoné y lógos a partir de lo que define, para Heidegger, el ser de los seres vivientes, su modo de ser-en-el-mundo.

En el análisis heideggeriano de la conceptualidad de estos conceptos, algunas conclusiones resultan de particular interés para comprender la posición de Heidegger en este seminario de 1924, pero también en relación con nuestros objetivos.

La primera, respecto de lo que debemos entender por mundo. No se trata, en efecto, de pensar la relación de los seres vivientes con el mundo según el modelo, ajeno al pensamiento griego, de la dicotomía entre un sujeto cognoscente y un objeto conocido. Ni el animal ni el hombre, en el análisis heideggeriano, son tomados como sujetos que primariamente conocen (sensitivamente los animales, intelectualmente los hombres), ni el mundo es una colección de objetos que esperan ser des- 
cubiertos. La relación de los seres vivientes, animales y hombres, con el mundo no es primariamente la de conocerlo, sino la de ser en él donde el mundo "es ahí mayormente (zumeist) en el modo de lo beneficioso y de lo perjudicial" (HEIDEGGER, 2002, p. 47).

La segunda observación concierne, según la formulación de Heidegger, a la politicidad o, al menos, relativa politicidad del animal: "los animales ya son, en cierto sentido, zoá politiká (animales políticos). Los seres humanos son solamente mâllon (más) zôon politikón que, por ejemplo, las abejas" (HEIDEGGER, 2002, p. 50). La razón de esta politicidad del animal radica, en este cursi, para Heidegger, en el hecho que también los animales son-con-otro.

La tercera observación es, de algún modo, recíproca de la anterior. Si los animales, en su animalidad, son en cierto sentido políticos; el hombre, en su politicidad, no deja de ser animal: "todas las posibilidades que poseen los animales están también en los humanos, no como unas al lado de otras, sino determinadas por la ousía del hombre en su manera de ser-en-el-mundo" (HEIDEGGER, 2002, p. 53).

La cuarta y última observación que nos interesa aquí es respecto de la posibilidad de ser-en-el-mundo que es, para el hombre, equi o co-originaria con el hablar. En efecto, no se trata sólo de la pólis, sino también de la oikía y, por ello, de lo que las reúne a ambas: la koinonía, el ser-con-otros, el comunicarse. (HEIDEGGER, 2002, p. 50).

Teniendo presente estas observaciones, podemos volver sobre el modo en que Heidegger analiza, en su conceptualidad, los conceptos aristotélicos de phoné y lógos. Ellos remiten a lo que caracteriza dos modos en los que el mundo está abierto o, si queremos, a dos modos en los que el mundo se vuelve Umwelt. El término utilizado por Heidegger es “Erschlossenheit”, apertura. En la phoné el mundo está abierto como hedú (placer) y lyperón (dolor); en el lógos, como sumphéron (útil), blaberón (perjudicial) y agathón (bueno). Para la determinación del sentido de cada uno de estos conceptos griegos, vale la pena subrayarlo, Heidegger se sirve fundamentalmente de la Retórica de Aristóteles. Desde esta perspectiva, Heidegger (2002, p. 61) sostiene:

\footnotetext{
"Hoy tenemos una noción primitiva del lenguaje o ninguna en absoluto. EI documento concreto de la originalidad de la visión griega es la entera Retórica. Hablar es deliberar acerca de lo que es propicio, hablar-con-unotro; lógos es el modo de ser de los seres humanos en su mundo, de tal manera que este ser es, en sí mismo, ser-con-un-otro. Esta koinonía no sólo
} 
está determinada por el lógos en sí mismo, sino también por el hecho de que el lógos es una deliberación en el ámbito del Cuidado (Besorgens)."

Es necesario no olvidar que en este abordaje de la retórica se trata, sobre todo, de un análisis del ser-en-el-mundo del hombre que es un ser-con-un-otro, porque, co-originariamente, posee lenguaje deliberativo. Por ello, no es de extrañarse que la retórica sea, para Heidegger, primordialmente, no una técnica sino una posibilidad, una dýnamis, y, más precisamente, la posibilidad de ver en cada caso lo que es conveniente en el ámbito de la deliberación, aquello de lo que se trata, lo que está en discusión (HEIDEGGER, 2002, p. 117).

Desde esta perspectiva, en la consideración del lógos retórico, del lógos deliberativo como modo de ser-en-el-mundo, Heidegger se ocupa del ejemplo, parádeigma, como es tradicional, junto con el entimema. Y como también es tradicional explotando el paralelismo entre las formas de mostrar de la dialéctica y las de la retórica: el entimema corresponde al silogismo deductivo y el ejemplo, a la epagogé (frecuentemente traducida por inducción).

Con un ejemplo (Beispiel), quiero ejemplificar (exemplifizierten) algo, para que quede claro no el caso particular del propio ejemplo, sino más bien en vistas a lo que es 'en general', kathólou. Kathólou no es la validez general, sino simplemente lo que es 'así en general'. (HEIDEGGER, 2002, p. 133)

Heidegger no se extiende mucho más sobre la cuestión del ejemplo. No es esto, en todo caso lo que nos interesa aquí; sino más bien, como ya lo hemos aclarado, el encuadre o la interpretación fenomenológica de la Retórica aristotélica, de la que el ejemplo es una de sus piezas centrales. En este contexto, ni el entimema ni el ejemplo son meras tácticas discursivas y, mucho menos, como sucederá en una parte de la retórica postaristotélica con el caso del ejemplo, un recurso estilístico en el curso de la exposición. Entimema y ejemplo son, sobre todo y primariamente, modos del lógos deliberativo que constituye, en su posibilidad más propia, el ser-en-elmundo del animal político.

\section{De la condición humana a la imaginación}

Hannah Arendt llega a la Universidad de Marburgo el semestre de invierno de 
1924-1925, es decir, el inmediatamente sucesivo al que tuvo lugar el curso de Heidegger dedicado, en gran medida, a la Retórica de Aristóteles. Como señala Theodore Kisiel, no sabemos si ella tuvo acceso a las notas que circulaban de este seminario de Heidegger, "pero su propia obra, en lo que sólo puede ser llamado la protopolítica de la condición humana, está profundamente marcada por la única aproximación fenomenológica a la pólis griega a la que ella estuvo efectivamente expuesta durante su estadía en Marburgo". (KISIEL, 2005, p. 153). En la perspectiva de Kisiel, el análisis arendtiano de la acción es un desarrollo del Mitwelt, del mundo interhumano de Heidegger. Es más, The Human Condition puede ser visto como una radicalización del plural, a veces utilizado por Heidegger, en la fórmula "ser-con-otro" (Miteinandersein) con la que caracteriza la facticidad humana. (KISIEL, 2005, p. 154).

En este contexto, Arendt vincula, quizá por primera vez, la tensión-oposición entre bíos y zoé -que domina el debate biopolítico- con los procesos de la Modernidad cuando explica que la idea de una "economía política" resulta, para la mentalidad griega, "una contradicción en sus términos". (ARENDT, 1998, p. 29)

En efecto, la zoé y el bíos pertenecen, para los griegos, a esferas separadas: respectivamente, a la oikía y a la pólis. $Y$ también son objeto de dos técnicas diferentes, la economía, la administración de la casa, que implica una relación entre desiguales, y la política, el gobierno de la ciudad, que, al contrario, se funda sobre la relación entre iguales.

La novedad de la política moderna, según la tesis de Hannah Arendt, consiste, en cambio, en que esta separación ha desaparecido: la zoé ha ingresado en la pólis y, por lo tanto, la administración de la vida biológica se ha convertido en una cuestión política. Surge así la economía política o, lo que al menos en cierto sentido termina siendo un sinónimo, la biopolítica (aunque Hannah Arendt no utilice este término).

Ahora bien, si retomamos la célebre definición aristotélica, en la existencia de la política, de la pólis diría el filósofo, siempre ha estado en cuestión la zoé. En este sentido, no hay pólis sin zoé. Ésta es, en términos clásicos, una condición necesaria. La condición suficiente, en cambio, está dada por el modo en el que ella tiene el lógos.

Reformularía, entonces, en estos términos la tesis de Hannah Arendt: con la Modernidad política o, al menos con una parte de ella, la que coincide con la forma- 
ción de los Estados nacionales y las teorías que le sirven de fundamento, la novedad fundamental no consiste simplemente en que la zoé ha ingresado en la pólis, sino también en que ha cambiado el modo por el cual el animal antropóforo posee el lenguaje.

En un contexto y con un alcance diferentes, sin relación directa con la obra de $\mathrm{H}$. Arendt, siguiendo además otra cadena argumentativa, creo que esta idea es la que sostiene también Agamben cuando en su arqueología del juramento, en el Sacramento del linguaggio (2008), afirma que la tesis de Foucault, según la cual el hombre es el animal en cuya política está en juego su vida, y su tesis, según la cual el hombre es el animal en cuyo lenguaje está en juego su propia vida, son inseparables y dependen la una de la otra (AGAMBEN, 2008b, p. 94). Política y lenguaje son, retomando ese concepto heideggeriano frecuentemente utilizado en su seminario de 1924, Gleichursprünliche, equi o co-originarios (HEIDEGGER, 2002, p. 128).

Considerando adecuadamente las cosas, esta lectura no contradice necesariamente la tesis de Hannah Arendt. Podría decirse, al contrario, que esta reformulación no hace más que retomar algunos conceptos fundamentales del pensamiento político de Hannah Arendt que, al menos a nuestro juicio, han sido no han sido suficientemente tenidos en cuenta.

Por un lado, el que concierne a la relación entre vida y lenguaje. En otros términos, el ingreso de la zoé en la pólis, se da "co-originariamente" con una modificación del estatuto del lenguaje en el orden de la vida política del hombre y, más específicamente, respecto de las funciones retóricas del lenguaje. Con la Modernidad, el animal se vuelve político, estableciendo una determinada relación con el lenguaje, diferente a la que Aristóteles concibe en el célebre pasaje de la Política apenas referido.

Pero, por otro lado, hay un segundo elemento que no ha sido suficientemente sopesado. En H. Arendt, esta capacidad deliberativa del lenguaje está estrechamente ligada al concepto con el que marca, en relación con el análisis de la facticidad humana, uno de los momentos más antiheideggerianos de su concepción política: la natalidad, en la que se enraízan todas las actividades humanas. Según las palabras de Arendt, "la natalidad, y no la mortalidad, puede ser la categoría central del pensamiento político" (ARENDT, 1998, p. 9). Por supuesto, sería erróneo hacer de la zoé y de la natalidad dos sinónimos, pero también, separarlos por completo. 
Arendt resume esta relación co-originaria entre vida y lenguaje, en estos términos:

\begin{abstract}
"Si la acción como comienzo corresponde al hecho de nacer, si es la realización de la condición humana de la natalidad, entonces el discurso corresponde al hecho de la distinción y es la realización de la condición humana de la pluralidad, es decir, de vivir como ser distinto y único entre iguales." (ARENDT, 1998, p. 196)
\end{abstract}

Por ello, no basta, para comprender en toda su amplitud el análisis de Arendt, limitarse a retomar la célebre formulación acerca del ingreso de la zoé en la pólis como especificidad de la política moderna; es necesario considerar, además, el modo en que la vida está siempre en relación con la política, co-originariamente con la discursividad. Propiamente hablando, en definitiva, la política se sitúa, para Arendt, en la tensión entre la igualdad-natalidad y la pluralidad-discurso. Surge, como frecuentemente insiste, entre los hombres, y no del hombre.

En este contexto, en efecto, debemos entender el sexto y último capítulo de The Human Condition que se ocupa, como indica el título, de la vita activa en la época moderna. La tesis central de Arendt es que si la zoé ha ingresado en la pólis es porque se ha dado una progresiva exclusión del obrar y del hacer, para dar lugar, así, a una primacía del trabajo (ARENDT, 1998, p. 320). La Modernidad es, en este sentido, retomando la expresión de Arendt, " la victoria del homo laborans ». Con la particularidad, señalada por Arendt al inicio de su trabajo, de que esta victoria del homo laborans define a la época moderna, pero no es suficiente para describir el mundo moderno (según la distinción establecida por Arendt entre época moderna, siglos XVI-XX, y mundo moderno, el que comienza con las primeras explosiones atómicas), que se caracteriza por ser una sociedad de trabajadores, pero en vías de convertirse en una sociedad sin trabajo (ARENDT, 1998, p. 5-6).

Esta situación, según el análisis de Arendt, es inversa a la experiencia de la pólis griega. En este marco, señala Arendt, criticando las traducciones latinas de zôon politikón por animal sociale y de zôon lógon échon por animal rationale, « ser político, vivir en la pólis, significaba que todo se decía mediante palabras y con persuasión y no con la fuerza y la violencia », « la preocupación de los ciudadanos era hablar entre sí ». Fuera de la pólis, los no-ciudadanos, los esclavos y los bárbaros, eran aneu lógou, sin discurso (ARENDT, 1998, p. 26-27).

Ahora bien, el trabajo de Kisiel antes mencionado plantea una cuestión fundamental respecto de los desarrollos políticos de Heidegger y Arendt; uno, como 
sabemos, re-direccionado hacia el nazismo y otro, hacia el republicanismo. A partir del análisis de la pólis griega a la luz de la Retórica aristotélica, Heidegger, en efecto, se dirigirá, primero, hacia la República de Platón, dando lugar a una concepción política centrada en los conceptos de líder del pueblo y guardianes del Estado y, luego, hacia el Antígona de Sófocles. Arendt, en cambio, se dirigirá hacia Kant (ARENDT, 1998, p. 149).

Nos encontraremos, así, a partir de un punto de partida común con dos maneras diferentes y también opuestas de abordar la relación de cada hombre con la esfera de lo público o, en términos heideggerianos, con el das Man (el "se" impersonal).

Ahora bien, es en este giro hacia Kant que nos encontramos, en Hannah Arendt, con la cuestión del ejemplo; en particular, como señalamos, en las lecciones de 1970 sobre la filosofía política de Kant y, más específicamente todavía, en las notas tituladas "Imagination".

En estas pocas y densas páginas, $\mathrm{H}$. Arendt busca mostrar que el lugar que ocupan los esquemas en la Kritik der reinen Vernuft, donde Kant describe las condiciones de posibilidad del conocimiento científico, es ocupado, en la Kritik der Urteilskraft, por el ejemplo. En segundo lugar, ella busca mostrar la relevancia de este concepto para la filosofía política.

¿Qué pasa, en efecto, cuando tenemos que pensar, formar juicios, pero no disponemos de un universal debajo del cual subsumir los particulares y, entonces, debemos descubrir o buscar la universalidad partiendo de los particulares? Para estos casos, Kant habla de juicios reflexivos, pues, precisamente, es por el trabajo de reflexión del entendimiento que podemos encontrar esta regla. En estos casos, también la imaginación desempeña una función fundamental, pero no a través de los esquemas, sino de los ejemplos.

El ejemplo es ese caso particular que la imaginación nos permite hacerlo nuevamente presente (para Kant, en efecto, ésta es la tarea de la imaginación y no de la memoria), "que contiene en sí, o se supone que contiene, un concepto o una regla general" (ARENDT, 1992, p. 84). Así, podemos pasar, retomando la formulación de H. Arendt, de Napoleón Bonaparte al bonapartismo. Esto es lo que sucede, señala, con muchos de nuestros conceptos históricos y de las ciencias políticas (ARENDT, 1992, p. 85). 


\section{A modo de conclusión}

Michel Foucault y Giorgio Agamben representan, como sabemos, dos de los autores que dominan el debate biopolítico contemporáneo. ${ }^{3}$ En cuanto concierne específicamente a la noción de ejemplo, para Agamben, como hemos señalado, ella define el método propio de la arqueología filosófica, en general, y de la arqueología de la biopolítica, en particular.

En otros dos trabajos, Agamben ha abordado con cierto detalle la noción de ejemplo. En La comunità che viene, lo hace en el tercer capítulo donde, a través del ejemplo, describe el ser de los miembros de esta comunidad. Ellos, como los ejemplos, escapan a la alternativa entre lo individual y lo universal (AGAMBEN, 1990, p. 14). Y en Homo sacer. Il potere sovrano e la nuda vita la noción de ejemplo es presentada como la contraparte de la noción de excepción, con la que forma un sistema. Mientras en la excepción, la vida está en relación con la ley en una posición de exclusión inclusiva (la vida está incluida en la medida en que está excluida, es decir, en cuanto vida desnuda), en el ejemplo, en cambio, nos encontramos con una relación de inclusión exclusiva. Un ejemplo es tal porque, excluido, muestra en su singularidad el ser de las otras singularidades que pertenecen a su clase, en la que está incluido (AGAMBEN, 1995, p. 26-27).

Foucault, a diferencia de Agamben, no ha prestado una particular atención a la noción de ejemplo. A pesar de ello, la idea de que la vida y su forma, el bíos, puedan ser pensadas en términos de ejemplaridad 0 , más precisamente, la idea de que la vida verdadera sea una forma de ejemplaridad aparece claramente en sus últimos cursos en el Collège de France. En este sentido, la vida de los cínicos, de los que se ocupa ampliamente en Le Courage de la vérité, es presentada como una vida ejemplar (FOUCAULT, 2009, p. 247).

Tanto en Foucault como en Agamben, la cuestión del ejemplo aparece, de este modo, a través de la necesidad de pensar, más allá de la ley y de la norma, la relación entre vida y lenguaje. El ser del ejemplo, sostiene explícitamente Agamben, es un "ser lingüístico" (AGAMBEM, 1990, p. 15). En cuanto a Foucault, la ejemplaridad de la vida se encuadra en el marco de lo que denomina una dramática del discurso (FOUCAULT, 2008, p. 66).

\footnotetext{
${ }^{3}$ De la noción de ejemplo en Agamben y Foucault nos hemos ocupado detalladamente en « Cuestiones de método: la problemática del ejemplo en Foucault y Agamben“, Res Publica, Madrid, en prensa.
} 
Resulta particularmente significativo, por ello, que en ambos casos, a diferencia de cuanto ocurre en los análisis de Martin Heidegger y Hannah Arendt, para pensar la relación inevitablemente política, entre vida y lenguaje, la retórica haya sido dejada de lado. En Agamben, por un lado, porque su elaboración del ejemplo se centra en los Analíticos primeros y, por otro, porque, en el desarrollo de la serie Homo sacer, la relación entre vida y lenguaje, se encamina, no hacia la problemática política de la deliberación, sino hacia la jurídico-religiosa del juramento, como lo muestra su trabajo del 2008 sobre /l sacramento del linguaggio (FOUCAULT, 2008, p. 66). En Foucault, la ausencia de la retórica se debe, en gran medida, al desarrollo interno de su pensamiento. Su dramática del discurso se ubica, en efecto, en el otro extremo de un recorrido iniciado con su primer curso, las Leçons sur la volonté de savoir, donde, para definir su práctica de la descripción enunciativa, sí se servía de la concepción retórica y sofística del lenguaje. En esta dramática del discurso, además, resulta necesario tenerlo presente, la noción central de parresia es abordada, sobre todo, como una forma de relación del sujeto consigo mismo, es decir, desde una perspectiva primariamente ética y no política.

Nos encontramos aquí con un desafío y, al mismo tiempo, con una necesidad. Pues, como ya hemos señalado, una de las tareas de la biopolítica es pensar la vida más allá de la biopolítica, es decir, más allá de los mecanismos y dispositivos que buscan sujetar la vida a la forma de la ley o de la norma. Ahora bien, esta tarea pasa inevitablemente por explorar las formas en que el hombre posee y usa el lenguaje, declinando así los sentidos posibles de la célebre definición aristotélica acerca del hombre como zôon lógon échon. En esta tarea, como puede seguirse de los análisis de Heidegger y Arendt, la concepción retórica del lenguaje y la Retórica de Aristóteles, en particular, con la centralidad de la noción de ejemplo, resultan decisivas a la hora de pensar el modo en que vida y lenguaje entran en esa relación constitutiva que hace posible la dimensión propiamente política del hombre y propiamente humana de la política.

Junto con una biopolítica de la norma, como la de Foucault, y una biopolítica de la ley, como la de Agamben en Homo sacer, e inevitablemente a partir de ellas, este desafío y esta necesidad pueden sintetizarse en la tarea de pensar una biopolítica de la ejemplaridad, que no puede no ser, por ello mismo, sino una biopolítica de la pluralidad. 


\section{REFERENCIAS}

AGAMBEN, Giorgio. La comunità che viene. Torino: Bollati Boringhieri, 1990.

AGAMBEN, Giorgio. Homo sacer. II potere sovrano e la nuda vita. Torino: Einaudi, 1995.

AGAMBEN, Giorgio. Signatura rerum. Sul método. Torino: Bollati Boringhieri, 2008a.

AGAMBEN, Giorgio. II sacramento del linguaggio. Una archeologia del giuramento. Bari: Laterza, 2008b.

ARENDT, Hannah. Lectures on Kant's Political Philosophy. Chicago: The University of Chicago Press, 1992.

ARENDT, Hannah. The Human Condition. Chicago-London: The University of Chicago Press, 1998.

CASTRO, Edgardo. Lecturas foucaulteanas. Una historia conceptual de la biopolítica. La Plata: Unipe Editorial Universitaria, 2011.

FERRARA, Alessandro. The Force of Example. Explorations in the Paradigm of Judgement. New York: Columbia University Press, 2008.

FOUCAULT, Michel. L’Archéologie du savoir. Paris: Gallimard, 1969.

FOUCAULT, Michel. Le Gouvernement de soi et des autres. Cours au Collège de France 1982-1983. Paris: Gallimard-Seuil, 2008.

FOUCAULT, Michel. Le Courage de la vérité. Le gouvernement de soi et des autres II. Cours au Collège de France 1983-1984. Paris: Gallimard-Seuil, 2009.

HARVEY, Irene. Labyrinths of Exemplarity. New York: State University of New York Press, 2002. 
HEIDEGGER, Martin. Sein und Zeit. Tübingen: Max Niemeyer, 1967.

HEIDEGGER, Martin. Ueber den Humanismus. Frankfurt: Klostermann, 2000.

HEIDEGGER, Martin. Grundbegriffe der aristotelischen Philosophie, Gesamtausgabe. V. XVIII. Frankfurt: Klostermann, 2002.

KISIEL, Theodore. The Genesis of Heidegger's Being and Time. Berkeley/Los Angeles: University of California Press, 1993.

KISIEL, Theodore. "Rhetorical Protopolitics in Heidegger and Arendt". In: GROSS, Daniel; KEMMANN, Ansgar (Ed.). Heidegger and Rhetoric. New York: State New York University Press, 2005. p. 153.

KRESS, John. Aristotle's Hermeneutics of Facticity: Heidegger's Early Phenomenological Interpretation of Aristotle. Research in Phenomenology, v. 36, n. 1, p. 329330, 2006.

Artigo:

Recebido em: 24/06/2012

Aceito em: 20/07/2012 